\section{Insegurança alimentar e estado nutricional de crianças de Gameleira, zona da mata do Nordeste brasileiro}

\section{Food insecurity and the nutritional status of children in Gameleira, in the Forest Zone of the Brazilian Northeast}

Juliana Souza Oliveira 1

Pedro Israel Cabral de Lira 2

Sandra Regina Maia 3

Leopoldina Augusta de Souza Sequeira 4

Roberta Corrêa de Araújo de Amorim 5

Malaquias Batista Filho 6

1 Centro Acadêmico de Vitória. Núcleo Nutrição da Universidade Federal de Pernambuco. Rua Alto do Reservatório, s.n. Bela Vista. Vitória de Santo Antão, PE, Brasil. CEP: 55608-680. E-mail: juliana_nutricao@yahoo.com.br

2-4 Departamento de Nutrição. Universidade Federal de Pernambuco. Recife, PE, Brasil.

5 Centro de Pesquisas Aggeu Magalhães (CPqAM/Fiocruz). Recife, PE, Brasil.

6 Instituto de Medicina Integral Prof ${ }^{\circ}$ Fernando Figueira. Recife, PE, Brasil.

\section{Resumo}

Objetivos: analisar a associação entre a insegurança alimentar das famílias e estado nutricional de menores de cinco anos.

Métodos: estudo transversal, de 501 famílias com 697 menores de cinco anos, no município da Gameleira, Pernambuco. A avaliação da (in)segurança alimentar foi realizada através da Escala Brasileira de Insegurança Alimentar (EBIA). A classificação do estado nutricional foi feita a partir dos indicadores estatura/idade, peso/idade e indice de massa corporal (IMC), utilizando o padrão de crescimento infantil da OMS. Foram estudados a associação de indicadores socioeconômicos, de insegurança alimentar e variáveis biológicas da criança sobre o indice estatura/idade, utilizando-se análise de regressão linear multivariada.

Resultados: a insegurança alimentar foi caracterizada em quase $90 \%$ das famílias, sendo a forma grave mais prevalente. Verificou-se uma prevalência baixa de déficit de peso pelo IMC e elevadas de déficit estatura/idade. O modelo final desta análise mostrou que as variáveis renda familiar per capita, escolaridade materna e idade da criança influenciaram significativamente o estado nutricional, entretanto, a EBIA não se associou com o estado nutricional das crianças.

Conclusões: é evidente a discrepância entre a prevalência de insegurança alimentar nas famílias e a frequência baixa de desnutrição nas crianças, expressando que os dois indicadores avaliam aspectos e situações bem diferenciadas.

Palavras-chave Estado nutricional, Criança,

Segurança alimentar e nutricional 


\section{Introdução}

Em termos de mercados globalizados a disponibilidade mundial de alimentos é superavitária, porém em muitos países e regiões em desenvolvimento, particularmente em relação aos grupos sociais e famílias de baixas condições socioeconômicas, o acesso regular e permanente a uma cesta básica de produtos suficiente e adequada para boa condição de saúde e nutrição mostra-se problemático, representando, assim, uma situação de insegurança. Objetivamente, estima-se que mais de 800 milhões de pessoas sobrevivem nesta condição. ${ }^{1}$ No entanto, subjetivamente, o contingente de famílias e seus membros que não se consideram seguros quanto ao acesso físico e econômico regular para o pleno atendimento de suas necessidades de consumo alimentar seria bem maior. ${ }^{2}$ Conceitualmente, esta condição se aplica às famílias que, em função de experiências e expectativas adversas, como instabilidade de emprego e geração de renda, se sentem sob risco de não dispor de recursos para a aquisição da cesta habitual de alimentos demandados pelo aglomerado familiar. ${ }^{3}$

A avaliação do consumo quali e quantitativo de alimentos e, sobretudo, do estado nutricional, pode ser realizada através de métodos relativamente bem estabelecidos. Todavia, quando se trata da insegurança alimentar, só na década de 90 nos Estados Unidos 4,5 e, mais recentemente, no Brasil 6 passaram a ser desenvolvidos modelos tentativos de avaliação dos aspectos subjetivos implícitos na situação das famílias potencialmente expostas às incertezas da provisão alimentar. Na realidade, em comparação com outros países ${ }^{7}$ o Brasil dispõe da experiência mais extensa e mais diversificada na avaliação da insegurança alimentar, contando, inclusive, com um modelo de questionário ajustado para diversas situações testadas no território nacional: a Escala Brasileira de Insegurança Alimentar (EBIA). ${ }^{6}$

Como instrumento de avaliação subjetiva, a escala EBIA tem sido validada em relação aos resultados de compra/acesso aos alimentos básicos da família. No entanto, por conta do próprio conceito de segurança alimentar/bem-estar nutricional, se estabelece a expectativa de uma desejável correspondência entre as duas condições, desde que a primeira torna-se pré-requisito para a segunda. Este duplo interesse é peculiarmente relevante em relação às crianças que representam, nas famílias de risco, o segmento biológico mais vulnerável à insegurança alimentar e suas consequências nutricionais mais imediatas e mais graves.

Neste artigo objetiva-se analisar a relação entre condição de segurança alimentar das famílias e estado nutricional de crianças menores de cinco anos de idade em uma localidade com acentuado grau de pobreza.

\section{Métodos}

O estudo, do tipo transversal, foi realizado entre março e maio de 2005, numa amostra de 501 famílias com 697 menores de cinco anos, (335 e 362 da zona urbana e rural, respectivamente) no município da Gameleira, na Zona da Mata Meridional de Pernambuco, em conglomerados escolhidos intencionalmente, a partir de dados obtidos junto à Secretaria Municipal de Saúde, segundo dois critérios: áreas de condições socioeconômicas precárias e existência de crianças menores de cinco anos, nas famílias.

Trata-se de um dos municípios mais pobres do Estado de Pernambuco, com uma história de três séculos de monocultura canavieira, estabelecendo um quadro social de penúria que se acentua na entressafra da cana, quando o desemprego rural e urbano pode atingir a metade ou mais de toda a população. Segundo o Instituto Brasileiro de Geografia e Estatística (IBGE), 62,8\% dos chefes de famílias não tinham renda monetária ou ganhavam apenas um salário mínimo. A taxa de mortalidade infantil era de 85 por 1000 nascidos vivos, incluindo-se entre as mais elevadas do Estado e do país. ${ }^{8}$

Para o cálculo da amostra levou-se em consideração uma prevalência de $60 \%$ de insegurança alimentar para o Nordeste, com base nos resultados da Pesquisa Nacional por Amostra de Domicílios 2004,7 estimando um erro máximo de $\pm 5 \%$, para um nível de significância de $95 \%$ que, acrescida de $10 \%$ para possíveis perdas, resultou uma amostra mínima de 440 famílias. Para o cálculo utilizou-se o programa STATCALC do EPI-INFO, versão 6.04

Para a coleta de dados foram utilizados um questionário estruturado contendo informações sobre os membros da família, as condições socioeconômicas, demográficas, características do domicílio, dados antropométricos e de saúde das crianças e a Escala Brasileira de Insegurança Alimentar (EBIA).

Fez-se um estudo em 30 famílias, objetivandose, além de testar o instrumento de coleta, experimentar e ajustar a logística do trabalho de campo. $\mathrm{O}$ acesso ao domicílio foi realizado com a concordância do chefe da família. Para este estudo, foi necessário o consentimento livre e esclarecido de cada um dos membros da família, incluindo o consentimento dos pais ou responsável para os 
menores de idade.

A avaliação da insegurança alimentar foi efetuada mediante a aplicação da Escala Brasileira de Insegurança Alimentar (EBIA) ${ }^{6}$ composta de 15 perguntas fechadas, com respostas positivas e negativas, relativas à percepção dos entrevistados sobre a situação alimentar vivida nos últimos três meses anteriores à entrevista. As respostas foram codificadas, considerando o processo de insegurança alimentar crescente, passando pela preocupação com a falta de alimentos, pelo comprometimento da qualidade da alimentação e, por último, pela deficiência quantitativa da alimentação e até mesmo fome entre adultos e crianças da família. Para as respostas positivas, foi atribuído o valor 1 (um), e para as negativas o valor 0 (zero), resultando num escore com amplitude de 0 a 15 pontos. A soma dos escores resultantes foi classificada em quatro níveis: 0 (zero), segurança alimentar; 1 a 5, como insegurança alimentar leve; 6 a 10, como insegurança alimentar moderada; e 11 a 15 , como insegurança alimentar grave.

A avaliação antropométrica foi realizada após treinamento dos pesquisadores segundo procedimentos técnicos recomendados pela Organização Mundial da Saúde, ${ }^{9}$ obtido utilizando balança digital (Filizola E-150/3P, São Paulo, Brasil) com capacidade para $150 \mathrm{~kg}$ e precisão de $100 \mathrm{~g}$. Crianças que ainda não andavam foram pesadas nos braços do responsável, cujo peso foi deduzido do peso total. Os menores de dois anos foram medidos em decúbito dorsal, com as pernas juntas e em extensão (tomada do comprimento), utilizando o infantômetro (Raven Equipment, made in England) com amplitude de 100 $\mathrm{cm}$ e subdivisões de $0,1 \mathrm{~cm}$. As crianças maiores de dois anos foram medidas em pé e descalças, utilizando-se estadiômetro (Cardiomed, CuritibaPR) com amplitude de $200 \mathrm{~cm}$ e subdivisões de $0,1 \mathrm{~cm}$.

Para garantir a acurácia das mensurações, as medidas do comprimento/altura foram tomadas duas vezes para cada criança com a condição de que a diferença entre as avaliações não deveria exceder 0,5 $\mathrm{cm}$. Se esse limite fosse ultrapassado, repetia-se a mensuração, anotando-se as duas medições com valores mais próximos, utilizando a média de cada medida para efeito de registro.

Os questionários foram compostos por perguntas pré-codificadas e checadas diariamente para a identificação de erros de preenchimento. Para a validação da digitação, foi realizada a dupla entrada de dado, através do programa Epi Info, versão 6.04 (CDC, Atlanta, USA). As análises estatísticas foram realizadas através do software Statistical Package for the Social Sciences, version 12.0.1 for Windows (SPSS Inc., Chicago, IL, USA). Para análise da consistência interna das variáveis, foi calculado o Alpha de Cronbach, aceitando-se como satisfatório um valor mínimo de $0,85.6$

$\mathrm{Na}$ avaliação do estado nutricional foi utilizado o software Anthro - 2007,10 sendo as crianças classificadas pelos índices, estatura/idade (E/I); peso/idade $(\mathrm{P} / \mathrm{I})$; peso/estatura $(\mathrm{P} / \mathrm{E})$ e índice de massa corporal (IMC), expressos em escore-Z. O padrão de crescimento infantil utilizado como referência para classificação das medidas de peso e estatura foi o da World Health Organization, 11 adotando-se os pontos de corte para categorização dos resultados: Desnutrição: < -2 escores Z; Risco nutricional: -2 escores $Z$ a $<-1$ escores $Z$; Adequado: -1 escore $Z$ a $<2$ escores $Z$; Sobrepeso: $\geq 2$ escores $Z$.

A variável dependente estatura/idade foi analisada como contínua. As correlações foram estimadas com referência a este índice, por apresentar maior a prevalência de déficit nutricional e ser considerada, atualmente, como a expressão epidemiológica mais característica e representativa de desvio antropométrico na população brasileira. O teste tStudent e a análise de variância (ANOVA) foram utilizados para comparar as diferenças entre médias nas análises bivariadas, considerando-se como significância estatística valores $p \leq 0,05$.

A matriz de correlação não identificou multicolinearidade entre as variáveis, visto que os coeficientes de correlação de Pearson foram inferiores a 0,18 . A análise de regressão linear múltipla foi realizada utilizando-se o modelo hierarquizado de entrada de variáveis, com a finalidade de avaliar o impacto das variáveis explanatórias sobre o índice estatura/idade. As variáveis explanatórias eram dicótomas, exceto a insegurança alimentar, que foi tratada como variável indicadora (dummy). Todas as variáveis com valor de $p<0,20$ na análise bivariada foram selecionadas para inclusão inicial na análise de regressão.

Adotou-se um processo de modelagem por blocos, utilizando-se o método de sequência "enter", de modo que, inicialmente o estado nutricional dos menores de cinco anos foi ajustado pela variável renda familiar per capita. O segundo bloco foi composto pelas variáveis, escolaridade materna e área geográfica. No terceiro bloco foi introduzida a variável relacionada à insegurança alimentar. Finalmente, no quarto bloco, incorporou-se a idade da criança. Foram considerados significantes valores de $p \leq 0,05$.

O projeto de estudo foi aprovado pelo Comitê de Ética do Instituto Materno Infantil Prof. Fernando 
Figueira (IMIP), atendendo às normas regulamentares de pesquisas envolvendo seres humanos Resolução 196/96, do Conselho Nacional de Saúde. Um médico pediatra fez parte da equipe de campo, com a responsabilidade de atender ou encaminhar para outros profissionais e serviços de assistência à saúde os casos clínicos que necessitassem de melhor exploração diagnóstica ou cuidados de acompanhamento.

\section{Resultados}

$\mathrm{O}$ instrumento para avaliar a insegurança alimentar revelou uma consistência interna satisfatória, visto que o Alpha de Cronbach foi de 0,93 .

Demonstra-se, na Figura 1, que aproximadamente $12 \%$ das famílias dos menores de cinco anos foram classificadas na categoria de segurança alimentar, predominando, assim, a condição de insegurança alimentar, sendo a forma grave a mais prevalente $(36,9 \%)$. No que se refere à localização, verifica-se que na área urbana prevaleceu a situação mais grave da insegurança alimentar $(43,8 \%)$ enquanto na zona rural predominou a forma moderada $(34,4 \%)$.

\section{Figura 1}

Prevalência da insegurança alimentar das famílias de menores de cinco anos nas zonas urbana e rural do município da Gameleira, PE, 2005.

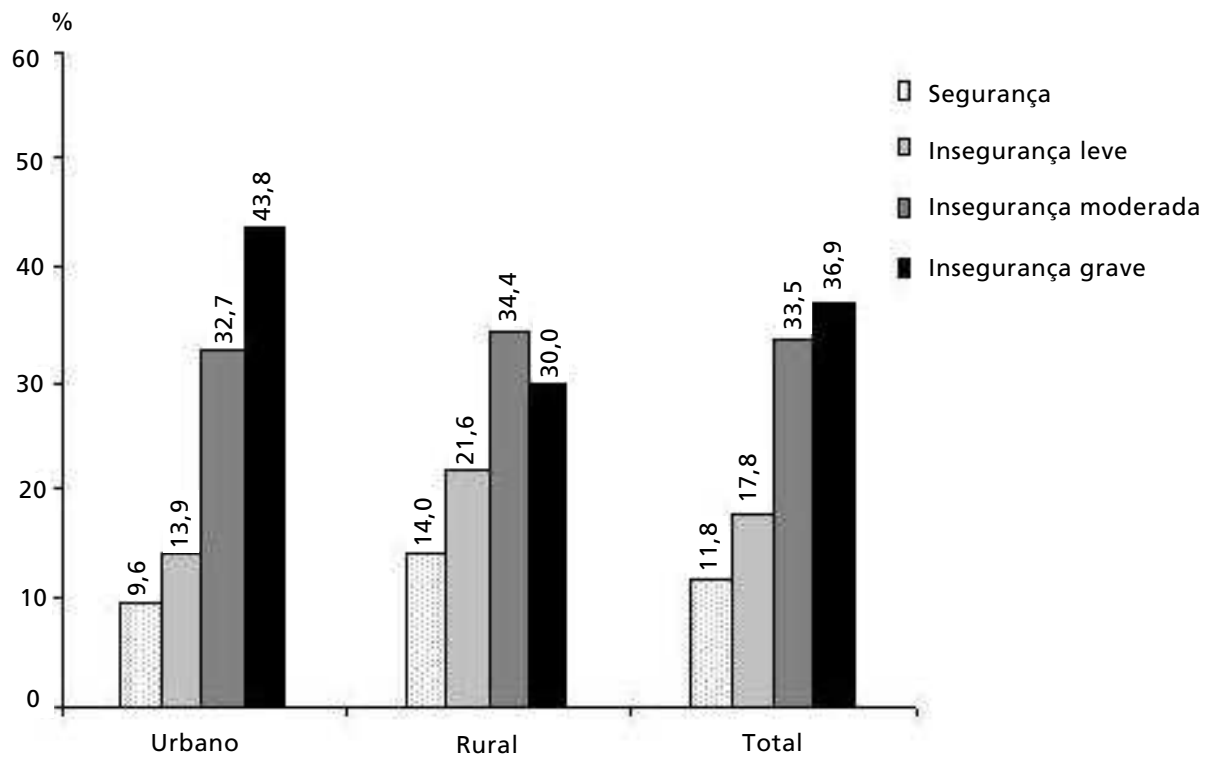

No que se refere ao estado nutricional das crianças, verificou-se uma prevalência muito baixa de desnutrição pelo o indicador peso/estatura e pelo índice de massa corporal (IMC) com valores de $2,7 \%$ e $2,4 \%$ na zona urbana, respectivamente e $1,9 \%$ na zona rural, para os dois índices, enquanto a frequência de adequação antropométrica ficou em torno dos $85 \%$. A prevalência de sobrepeso da zona rural foi quase duas vezes maior que no meio urbano. Com relação ao índice peso/idade, obtevese uma prevalência de desnutrição de $5,7 \%$ na zona urbana e $4,7 \%$ no meio rural. Por fim, de acordo com o índice estatura/idade, foram encontradas frequências elevadas de desnutrição: respectivamente $14,9 \%$ e $17,9 \%$, nas áreas urbana e rural (Figura 2).

Como se observa na Tabela $1,75,1 \%$ dos menores de cinco anos se encontravam em famílias na condição de insegurança alimentar moderada ou grave. A insegurança alimentar, em seus diferentes graus de intensidade, quando associada ao índice estatura/idade, demonstrou uma relação estatistica- 
mente significante, com a média de escores $\mathrm{Z}$ diminuindo à medida que a insegurança alimentar se agravava. No que diz respeito à localização, nota-se que a média de escores $\mathrm{Z}$ foi menor na zona rural, sendo a diferença estatisticamente significante. Verifica-se que mais de $90 \%$ das famílias percebiam renda familiar per capita abaixo da linha de pobreza (menos de 0,5 salários mínimos per capita/mês) e que, quando relacionada ao índice estatura/idade, resultou numa associação estatisticamente signifi- cante. As mães apresentavam baixo nível de escolaridade, visto que $67,8 \%$ tinham até quatro anos de estudo. À medida que aumentavam os anos de escolaridade materna, aumentava-se a média de escore Z Quanto ao sexo, não houve diferença estatisticamente significante nas médias de escores $\mathrm{Z}$ e com relação à idade das crianças verificou-se que aquelas com dois anos ou mais tinham uma menor média de escores $Z$, com associação estatística.

Figura 2

Estado nutricional de menores de cinco anos das zonas urbano e rural de acordo com os indicadores peso/idade (P/I), estatura/idade (E/I), peso/estatura (P/E) e índice de massa corporal (IMC). Gameleira, PE, 2005.

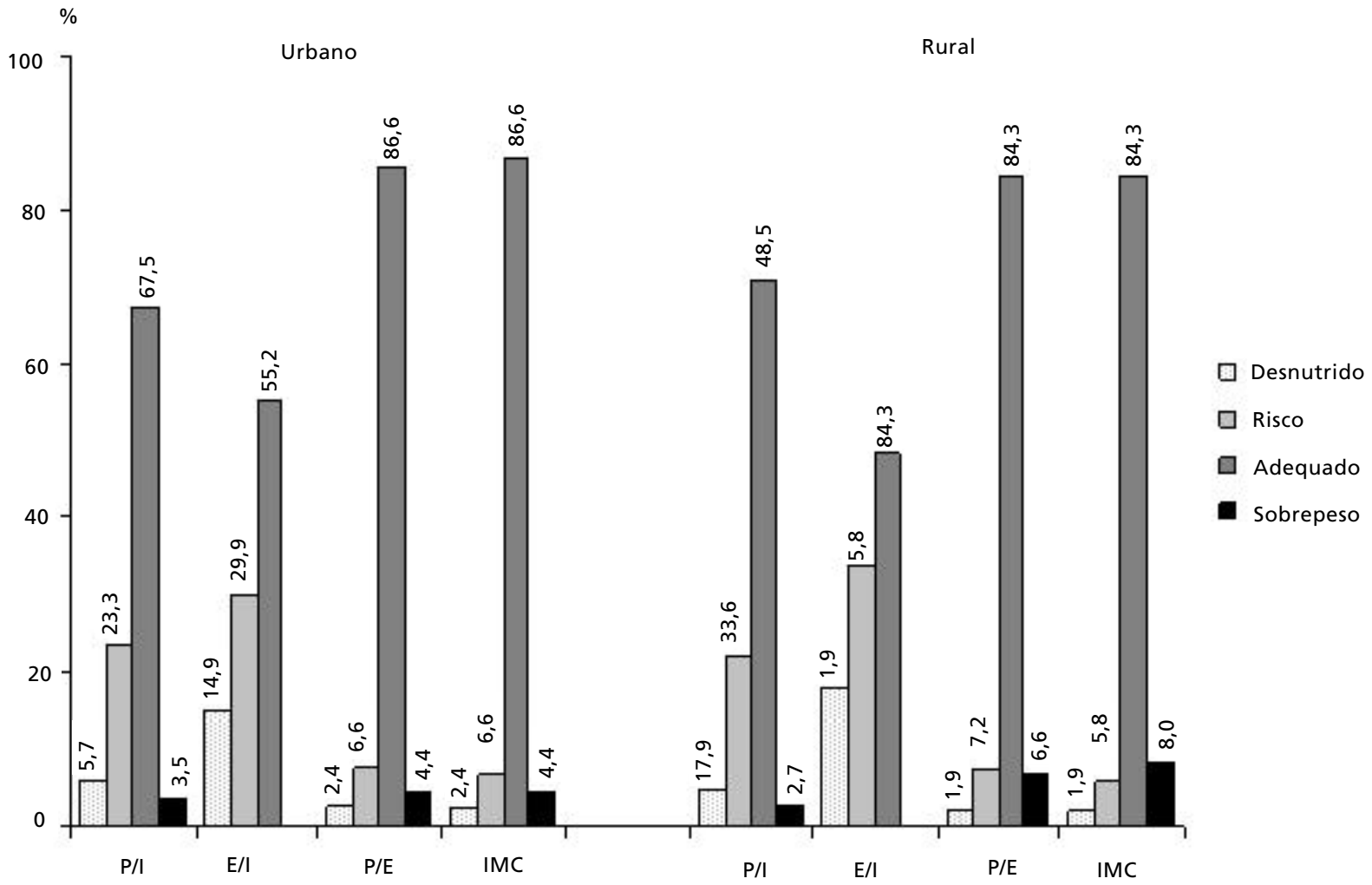

A análise dos efeitos combinados das variáveis explanatórias em relação ao índice estatura/idade encontra-se na Tabela 2. O modelo 1 revela que a renda familiar per capita influenciou significativamente os resultados deste indicador. O modelo 2 mostra que a escolaridade materna permaneceu significante após ajuste para a variável renda familiar per capita, enquanto o papel da localização (urbana $x$ rural) deixou de ser estatisticamente significante. No modelo 3 , o efeito da insegurança alimentar não apresentou significância estatística após ajuste para as variáveis dos blocos 1 e 2 . Por fim, a entrada da idade, no modelo 4 , revelou-se significante com a resposta do estado nutricional, após ajuste para as demais variáveis. 


\section{Tabela 1}

Índice estatura/idade, expresso em escore $Z$, de menores de cinco anos, segundo variáveis socioeconômicas, biológicas e outras características. Gameleira, PE, 2005.

\begin{tabular}{|c|c|c|c|c|c|c|}
\hline \multirow[t]{2}{*}{ Variáveis } & \multicolumn{5}{|c|}{ Estatura/Idade } & \multirow[t]{2}{*}{$p$} \\
\hline & $\mathrm{n}$ & $\%$ & $\bar{x}$ & $\mathrm{DP}$ & IC95\% & \\
\hline \multicolumn{7}{|l|}{ Insegurança alimentar } \\
\hline Segurança Alimentar & 66 & 9,5 & $-0,51$ & 1,31 & $-0,83$ a $-0,19$ & 0,01 \\
\hline IA leve & 107 & 15,4 & $-0,82$ & 1,27 & $-1,07$ a $-0,58$ & \\
\hline IA moderada & 233 & 33,4 & $-0,88$ & 1,28 & $-1,05$ a $-0,72$ & \\
\hline IA grave & 291 & 41,7 & $-1,06$ & 1,17 & $-1,20$ a $-0,93$ & \\
\hline \multicolumn{7}{|l|}{ Área geográfica } \\
\hline Zona Urbana & 335 & 48,1 & $-0,80$ & 1,23 & $-0,93$ a $-0,67$ & 0,03 \\
\hline Zona Rural & 363 & 51,9 & $-1,01$ & 1,25 & $-1,14$ a $-0,88$ & \\
\hline \multicolumn{7}{|l|}{ Renda per capita (SM) } \\
\hline$<0,25$ & 449 & 64,3 & $-1,05$ & 1,20 & $-1,16$ a $-0,93$ & 0,01 \\
\hline $0,25-0,49$ & 198 & 28,4 & $-0,71$ & 1,33 & $-0,90$ a $-0,53$ & \\
\hline $0,50-0,74$ & 30 & 4,3 & $-0,70$ & 0,95 & $-1,05$ a $-0,34$ & \\
\hline$\geq 0,75$ & 21 & 3,0 & $-0,20$ & 1,29 & $-0,79$ a $-0,39$ & \\
\hline \multicolumn{7}{|l|}{ Escolaridade materna } \\
\hline Nunca frequentou escola & 96 & 13,8 & $-1,36$ & 1,21 & $-1,60$ a $-1,11$ & 0,01 \\
\hline $1-4$ anos & 377 & 54,0 & $-1,02$ & 1,20 & $-1,14$ a $-0,90$ & \\
\hline$>4$ anos & 225 & 32,2 & $-0,53$ & 1,24 & $-0,69$ a $-0,38$ & \\
\hline \multicolumn{7}{|l|}{ Sexo } \\
\hline Masculino & 345 & 50,6 & $-0,90$ & 1,31 & $-1,04$ a $-0,76$ & 0,81 \\
\hline Feminino & 353 & 49,4 & $-0,96$ & 1,22 & $-1,05$ a $-0,80$ & \\
\hline \multicolumn{7}{|l|}{ Idade } \\
\hline$<24$ meses & 277 & 39,7 & $-0,60$ & 1,10 & $-0,77$ a $-0,44$ & 0,01 \\
\hline$\geq 24$ meses & 421 & 60,3 & $-1,11$ & 1,09 & $-1,22$ a $-1,01$ & \\
\hline
\end{tabular}

SM=salário mínimo; $I A=$ Insegurança alimentar.

\section{Tabela 2}

Modelo de regressão linear hierarquizado de fatores determinantes do índice estatura/idade de menores de cinco anos. Gameleira, PE, 2005.

\begin{tabular}{|c|c|c|c|c|}
\hline Variáveis & Modelo $1 \beta$ & Modelo $2 \boldsymbol{\beta}$ & Modelo $3 \boldsymbol{\beta}$ & Modelo $4 \beta$ \\
\hline \multicolumn{5}{|c|}{ Renda familiar per capita (SM) } \\
\hline$<0,25$ & $-0,38 * *$ & $-0,28 * *$ & $-0,23^{*}$ & $-0,27^{*}$ \\
\hline \multicolumn{5}{|c|}{ Escolaridade Materna1 (anos) } \\
\hline $0-4$ & & $-0,47 * *$ & $-0,45 * *$ & $-0,39 * *$ \\
\hline \multicolumn{5}{|l|}{ Área geográfica1 } \\
\hline Rural & & $-0,14$ & $-0,16$ & $-0,14$ \\
\hline \multicolumn{5}{|c|}{ Insegurança Alimentar² } \\
\hline IA Leve & & & $-0,31$ & $-0,27$ \\
\hline IA Moderada & & & $-0,28$ & $-0,22$ \\
\hline IA Grave & & & $-0,32$ & $-0,26$ \\
\hline \multicolumn{5}{|l|}{ Idade 3} \\
\hline$\geq 24$ meses & & & & $-0,45 * *$ \\
\hline
\end{tabular}

SM=salário mínimo; IA=Insegurança alimentar; $\beta=$ Coeficiente de regressão; ${ }^{1}$ Ajustado pela variável renda familiar per capita; 2 Ajustado pela variável renda familiar per capita, escolaridade materna e área geográfica; 3 Ajustado pela variável renda familiar per capita, escolaridade materna, área geográfica e Insegurança alimentar; Níveis de significância: * $p \leq 0,05 ; * * p \leq 0,01$ 


\section{Discusssão}

Considera-se, inicialmente, que os resultados achamse validados por uma consistência interna alta, Alpha de Cronbach de 0,93, assegurando que as perguntas foram respondidas de acordo com a expectativa teórica própria da escala, em ordem crescente de gravidade específica de cada pergunta.

No que se refere a um dos objetivos básicos do estudo (a avaliação da insegurança alimentar em uma localidade caracteristicamente pobre, com um IDH dos mais baixos da mesorregião da zona da Mata de Pernambuco) os resultados indicam $88 \%$ das famílias com crianças menores de cinco anos se enquadravam nas diversas categorias de insegurança, com marcante predomínio das formas moderada e grave. No meio urbano, inclusive, menos de $10 \%$ das famílias foram classificadas na condição de segurança. A prevalência de insegurança alimentar praticamente generalizada comprova a adequação da escolha deste município para retratar uma situação de marcante pobreza de sua população, no contexto do Estado e do próprio país, tornando-se, portanto, um interessante estudo de caso para os objetivos do estudo. Trata-se, portanto, de uma situação bem mais desfavorável que a encontrada no conjunto de resultados do Nordeste, segundo a Pesquisa Nacional por Amostra de Domicílios - 2004 (PNAD) 7 e, mais recentemente, a Pesquisa Nacional de Demografia em Saúde - 2006 (PNDS), 12 nas quais, respectivamente, $59,1 \%$ e $52,9 \%$ das famílias foram enquadradas na categoria de insegurança. Por outro lado, na extensa e recente experiência brasileira sobre este objeto de estudo, apenas algumas situações publicadas em veículos de divulgação científica - Yuyama et al.,13 em famílias urbanas e rurais do Amazonas, Pereira et al.,14 caracterizando uma localidade de alta vulnerabilidade social da cidade de São Paulo e Oliveira et al. 15 estudando as famílias de crianças de São João do Tigre, no semiárido do Nordeste - encontraram resultados semelhantes aos aqui relatados. Observa-se ainda, como um resultado peculiar, a predominância de insegurança e, sobretudo, de formas graves (praticamente 44\%) no meio urbano, influenciadas pelas características próprias do seu perfil de consumo, podendo também representar uma manifestação singular do chamado "inchaço urbano". Trata-se do crescimento periférico das cidades por migrações oriundas do campo, aglomerando famílias que não encontram, no novo espaço de residência, condições de produzir seus próprios alimentos ou de comprá-los com a regularidade, quantidade e qualidade adequadas, pela própria instabilidade do emprego avulso e da renda aleatória nos espaços de pobreza das cidades "inchadas"16 pelo deslocamento rural/urbano. Esta situação se difunde como um processo ambivalente da modernidade e pobreza, em grande parte do mundo em desenvolvimento. ${ }^{17}$

Por outra parte, a situação nutricional das crianças também revela um quadro epidemiológico que repete o cenário dominante na Região Nordeste e no próprio país: o desaparecimento da desnutrição pelo índice peso/altura nas últimas décadas, principalmente pela redução das doenças prevalentes na infância, pela redução considerável da desnutrição energético-protéica (DEP) expressa pelo índice peso/idade e, caracteristicamente, a prevalência ainda bastante elevada de déficit estatural ou desnutrição pregressa, representada pelo índice estatura/idade. A este propósito, deve-se observar que a ocorrência do retardo estatural (14,9\% no meio urbano e $17,9 \%$ no rural) praticamente duplica as frequências relatadas em estudos recentes na Região Nordeste, 7,12 significando que, de fato, o município da Gameleira representa um espaço diferenciado de riscos nutricionais muito elevados. Ademais, conforme foi relacionado na abordagem metodológica, os setores de amostragem foram deliberadamente escolhidos para incluir os conglomerados reconhecidamente mais pobres do município. Sem dúvida, a baixíssima prevalência da desnutrição nas crianças pelo índice peso/estatura constitui um resultado que merece investigação, como já assinalaram outros estudos no Brasil,18,19 inclusive recorrendo a interpretações de possíveis efeitos do chamado crescimento alométrico ou crescimento diferencial, 20 já muito usado no campo da biologia (plantas e animais). É uma hipótese que começa a ser estudada na antropometria nutricional.

No entanto, o que deve ser destacado como foco central da análise é a grande discordância entre os resultados descritivos dos dois objetos de estudo: a insegurança alimentar praticamente generalizada (quase $90 \%$ das famílias), a situação praticamente controlada da desnutrição pelo índice de massa corporal (IMC) e pela relação peso/idade e, comparativamente, as grandes diferenças de prevalência quando confrontada com a ocorrência de insegurança alimentar. Desta forma, embora a relação estatística seja significativa na análise bivariada, a participação da insegurança alimentar, mesmo em sua forma grave, não se manifesta estatisticamente como fator de variação dos resultados do estado nutricional das crianças, na análise de regressão linear multivariada. Não figura, portanto, como um fator independente de risco, ao contrário da renda per capita abaixo de 0,25 salário-mínimo e da baixa 
escolaridade materna (0-4 anos de estudos), que se mantêm na sucessão de modelos explicativos do déficit estatural das crianças, esses resultados aqui encontrados se assemelham aos achados de Oliveira et al. 15 Por sinal, se estabelece uma razão elementar de lógica matemática: sendo a insegurança alimentar praticamente uma generalidade, na medida em que afeta quase $90 \%$ de toda a amostra, evidentemente extrapola a particularidade que seria a desnutrição, mesmo representada por seu indicador mais frequente, o retardo estatural, presente entre $15 \% \mathrm{e}$ $18 \%$ das crianças, respectivamente, no meio urbano e rural. E mesmo quando se restringe a análise estatística para a situação de insegurança grave, a esperada associação com o estado de nutrição não é encontrada no modelo de regressão hierarquizada.

$\mathrm{Na}$ realidade, a insegurança alimentar, como evento de caráter subjetivo, tem sua lógica própria: a insegurança de emprego, de renda, de instabilidade familiar, as dúvidas sobre a rede de proteção social e da solidariedade comunitária. ${ }^{21}$ Ademais, destaca-se o fato de que as famílias da amostra, necessariamente, com crianças menores de cinco anos, pode influir nos resultados da insegurança alimentar em função do temor da falta de alimentos das mães em relação a seus filhos. Fato que seria mais explicado no campo da psicologia social e da antropologia cultural e menos pela abordagem da antropologia física, ou seja, em relação aos indicadores somatométricos aqui utilizados para a avaliação do estado nutricional das crianças.

\section{Referências}

1. Organización de las Naciones Unidas para la Agricultura y la Alimentación (FAO). El estado de la inseguridad alimentaria en el mundo 2006 - La erradicación del hambre en el mundo: evaluación de la situación diez años después de la Cumbre Mundial sobre la Alimentación. Roma, 2006.

2. Melgar-Quiñonez H, Zubieta AC, Valdez E, Whitelaw B Kaiser L. Validación de un instrumento para vigilar la inseguridad alimentaria en la Sierra de Manantlán, Jalisco. Salud Pública de Méx. 2005; 47: 413-22.

3. Panigassi G. Inquérito populacional sobre a percepção da segurança alimentar intrafamiliar no município de Campinas, SP [tese]. Campinas: Faculdade de Ciências Médicas da Universidade Estadual de Campinas; 2005. [2007 Nov 01] Disponível em: http://libdigi.unicamp.br/document/?code=vtls000389627

4. Radimer KL, Olson CM, Greene JC, Campbell CC, Habicht JP. Understanding hunger and developing indicators to assess it in women and children. J Nutr Educ. 1992; 24: 3645 .
Estes aparentes desencontros de resultados podem, em grande parte, serem entendidos na análise conceitual elaborada por Monteiro, 22 estabelecendo diferenças e aproximações entre fome, pobreza e desnutrição. Ou o paradoxo de se encontrar elevadas prevalências de normalidade antropométrica, inclusive, com frequências superiores às obtidas nos países mais desenvolvidos, em espaços geográficos sob condições socioeconômicas caracteristicamente desfavoráveis, como o Nordeste Brasileiro, refletindo um momento peculiar do processo de transição nutricional. ${ }^{19}$ É neste sentido que devem ser considerados os diferentes indicadores da situação alimentar e nutricional, de tal modo que sua interpretação ressalta mais o caráter de complementaridade, de múltiplas leituras de diferentes aspectos do que suas discrepâncias, como nos desencontros entre os resultados da escala EBIA, por um lado, e a antropometria nutricional, por outro.

\section{Agradecimentos}

Ao $\mathrm{CNPq} / \mathrm{MCT} /$ Agronegócio pelo financiamento dos projetos e pela Bolsa de Produtividade em Pesquisa dos Profs. Pedro Israel Cabral de Lira e Malaquias Batista Filho, aos técnicos da Prefeitura da cidade da Gameleira-PE, a equipe de campo e todas às famílias pelo apoio e colaboração.

5. Wehlerr CA, Scott RI, Anderson JJ. The Community Childhood identification Project: a model of domestic hunger-demonstration project in Seattle, Washington. J Nutr Educ. 1992; 24: 29-35.

6. Perez-Escamilla R, Segall-Corrêa AM, Maranha LK, Sampaio MFA, Marín-León L, Panigassi G. An adapted version of the U.S. Department of Agriculture Food Insecurity Module is a valid tool for assessing household food insecurity in Campinas, Brazil. J Nutr. 2004; 135 : 1923-8

7. Instituto Brasileiro de Geografia e Estatísticas (IBGE). Pesquisa Nacional por Amostra de Domicílios. Segurança Alimentar: 2004. Rio de Janeiro; 2006.

8. Cavalcanti C, Dias A, Lubambo C, Barros H, Cruz L, Araújo MLC, Moreira M, Galindo O. Trabalhos para discussão. PROMATA - Programa de apoio ao desenvolvimento sustentável da Zona da Mata de Pernambuco. Fevereiro; 2002. 
9. World Health Organization (WHO). Physical Status: The use and interpretation of anthropometry. Technical Report Series, 854. Geneva; 1995.

10. World Health Organization (WHO). Anthro for personal computers, version 2, 2007: Software for assessing growth and development of the world's children. Geneva; 2007. [2007 Jan 23]. Disponível em: http://www.who.int/childgrowth/software/en/

11. World Health Organization (WHO). Multicentre Growth Reference Study Group. WHO child growth standards based on length/height, weight and age. Acta Paediatr. 2006; (suppl 450): 76-85.

12. Brasil. Ministério da Saúde (MS). PNDS 2006 - Pesquisa Nacional de Demografia e Saúde da Criança e da Mulher. Relatório. Brasília, 2008

13. Yuyama LKO, Aguiar JPL, Pantoja L, Maeda RN, Melo T, Alencar FH, Nascimento AMM, Negreiros NMA, SegallCorrêa AM, Pérez-Escamilla R. Segurança Alimentar/insegurança alimentar em famílias urbanas e rurais no estado do Amazonas: I. Validação de metodologia e de instrumento de coleta de informação. Acta Amaz. 2007; 37: 247-52.

14. Pereira DA, Vieira VL, Fiore EG, Cervato-Mancuso AM. Insegurança alimentar em região de alta vulnerabilidade social da cidade de São Paulo. Segurança Alimentar e Nutricional, Campinas. 2006; 13: 34-42.

15. Oliveira JS, Lira PIC, Andrade SLLS, Sales AC, Maia SR, Batista Filho M. Insegurança Alimentar e estado nutricional de crianças de São João do Tigre, no semiárido do Nordeste. Rev Bras Epidemiol. 2009; 12: 413-23.
16. Caballero B. Subnutrição e obesidade em países em desenvolvimento. Cadernos de Estudos Desenvolvimento Social em Debate, Brasília, DF: Ministério do desenvolvimento Social e Combate à fome, Secretaria de Avaliação e Gestão da Informção. 2005; 2 (1):10-13.

17. Sales T, Baeninger R. Migrações Internas e Internacionais no Brasil. Travessia (São Paulo). 2000; 36:33-44.

18. Post CL, Victora CG, Barros AJ. Low prevalence of bodyweight-for-height deficit: comparison of stunned and nostunned Brazilian children. Rev Saúde Pública. 1999; 33 575-85.

19. Batista Filho M, Miglioli TC, Santos MC. Normalidade antropométrica de adultos: o paradoxo geográfico e socioeconômico da transição nutricional no Brasil. Rev Bras Saúde Matern Infant. 2007, 7: 487-93.

20. Santos FRJB. Crescimento alométrico em crianças eutróficas e desnutridas [dissertação]. Recife: Instituto Materno Infantil de Pernambuco; 2000. 64 pp. Cad Saúde Pública. 2001, 17: 739-41.

21. Freitas MCS, Pena PGL. Segurança alimentar e nutricional: a produção do conhecimento com ênfase nos aspecto da cultura. Rev Nutr. 2007; 20: 69-81.

22. Monteiro CA. A dimensão da pobreza, da desnutrição e da fome no Brasil. Estud Av. 2003; 48:7-20.

Recebido em 27 de outubro de 2009

Versão final apresentada em 22 de abril de 2010

Aprovado em 4 de maio de 2010 